\title{
Skew Category Algebras Associated with Partially Defined Dynamical Systems
}

\author{
Patrik Lundström* Johan Öinert ${ }^{\dagger}$
}

\begin{abstract}
We introduce partially defined dynamical systems defined on a topological space. To each such system we associate a functor $s$ from a category $G$ to Top $^{\text {op }}$ and show that it defines what we call a skew category algebra $A \rtimes^{\sigma} G$. We study the connection between topological freeness of $s$ and, on the one hand, ideal properties of $A \rtimes^{\sigma} G$ and, on the other hand, maximal commutativity of $A$ in $A \rtimes^{\sigma} G$. In particular, we show that if $G$ is a groupoid and for each $e \in \operatorname{ob}(G)$ the group of all morphisms $e \rightarrow e$ is countable and the topological space $s(e)$ is Tychonoff and Baire, then the following assertions are equivalent: (i) $s$ is topologically free; (ii) $A$ has the ideal intersection property, that is if $I$ is a nonzero ideal of $A \rtimes^{\sigma} G$, then $I \cap A \neq\{0\}$; (iii) the ring $A$ is a maximal abelian complex subalgebra of $A \rtimes^{\sigma} G$. Thereby, we generalize a result by Svensson, Silvestrov and de Jeu from the additive group of integers to a large class of groupoids.
\end{abstract}

MSC2000: 16W50, 16S99

\section{Introduction}

We have known for a long time that there is a connection between topological properties of spaces and algebraical properties of rings. In the classical papers [12, [13] and [14] by von Neumann and Murray a link between ergodic theory and the theory of von Neumann algebras was established. Namely, they show that if the action is free, then the associated crossed product is a factor if and only if the action is ergodic. They also give precise conditions on the measure-theoretic side under which it is a factor of a certain type. A similar well-known result is the theorem of Krieger (see [7] and [8]) saying that two such ergodic group actions are orbit equivalent if and only if their associated crossed product von Neumann algebras are isomorphic. This strong ergodic interplay has stimulated the

*Address: University West, Department of Engineering Science, SE-46186 Trollhättan, Sweden, E-mail: Patrik.Lundstrom@hv.se, Fax: +46-520-223099

${ }^{\dagger}$ Address: Department of Mathematical Sciences, University of Copenhagen, Universitetsparken 5, DK-2100 Copenhagen Ø, Denmark, E-mail: oinert@math.ku.dk, Fax: +45-35320704 


\section{Introduction}

study of a more general topological situation in the following sense. Suppose that $X$ is a topological space and $s: X \rightarrow X$ is a continuous function; in that case the pair $(X, s)$ is called a topological dynamical system. An element $x \in X$ is called periodic if there is a positive integer $n$ such that $s^{n}(x)=x$; an element of $X$ which is not periodic is called aperiodic. Recall that the topological dynamical system $(X, s)$ is called topologically free if the set of aperiodic elements of $X$ is dense in $X$. Note that if $X$ carries the discrete topology, then $(X, s)$ is topologically free if and only if $(X, s)$ is algebraically free, that is if for every $x \in X$ and any positive integer $n$ we have that $s^{n}(x) \neq x$. Now suppose that $s$ is a homeomorphism of a compact and Hausdorff topological space $X$. Denote by $C(X)$ the unital $C^{*}$-algebra of continuous complex-valued functions on $X$ endowed with the supremum norm, conjugation as involution and pointwise addition and multiplication. The map $\sigma_{s}: C(X) \rightarrow C(X)$ which to a function $f \in C(X)$ associates $f \circ s \in C(X)$ is then an automorphism of $C(X)$. The action of $\sigma_{s}$ on $C(X)$ extends in a unique way to a strongly continuous representation $\sigma: \mathbb{Z} \rightarrow \operatorname{Aut}(C(X))$ subject to the condition that $\sigma(1)=\sigma_{s}$, namely $\sigma(k)=\sigma_{s}^{k}$, for $k \in \mathbb{Z}$. In that case, the associated transformation group $C^{*}$-algebra $C^{*}(X, s)$ can be constructed (see e.g. [2] or [17] for the details). It has been observed, in the works of Zeller-Meier [24], Effros, Hahn [3], Elliott [4], Archbold, Quigg, Spielberg [1, 18, 19], Kishimoto, Kawamura, Tomiyama [6, 5, 23], that topological freness of $(X, s)$ is closely linked with, on the one hand, ideal properties of $C^{*}(X, s)$ and, on the other hand, commutativity properties of $C(X)$ in $C^{*}(X, s)$. The main impetus for this article is the following elegant theorem (formulated as Theorem 4.5 in the book [23. by Tomiyama) summarizing such results from [1, 4, 5, 6, 23, 24].

Theorem 1. If $s$ is a homemorphism of a compact and Hausdorff topological space $X$, then the following assertions are equivalent:

(i) $(X, s)$ is topologically free;

(ii) if $I$ is a nonzero closed ideal of $C^{*}(X, s)$, then $I \cap C(X) \neq\{0\}$;

(iii) $C(X)$ is a maximal abelian $C^{*}$-subalgebra of $C^{*}(X, s)$.

Inspired by Theorem 1. Svensson, Silvestrov and de Jeu [22] have shown an analogous result (see Theorem 21) relating properties of an arbitrary topological dynamical system $(X, s)$, where $s$ is a homeomorphism, to ideal properties of the skew group algebra $C(X) \rtimes^{\sigma} \mathbb{Z}$ (note that in the literature this algebra is often referred to as a crossed product). Recall that $C(X) \rtimes^{\sigma} \mathbb{Z}$ is defined as the collection of formal sums $\sum_{n \in \mathbb{Z}} f_{n} u_{n}$, for $f_{n} \in C(X)$, where $f_{n}=0$ for all but finitely many $n \in \mathbb{Z}$. Define addition and multiplication on $C(X) \rtimes^{\sigma} \mathbb{Z}$ by

$$
\left(\sum_{n \in \mathbb{Z}} f_{n} u_{n}\right)+\left(\sum_{n \in \mathbb{Z}} g_{n} u_{n}\right)=\sum_{n \in \mathbb{Z}}\left(f_{n}+g_{n}\right) u_{n}
$$

respectively

$$
\left(\sum_{n \in \mathbb{Z}} f_{n} u_{n}\right)\left(\sum_{n \in \mathbb{Z}} g_{n} u_{n}\right)=\sum_{n \in \mathbb{Z}}\left(\sum_{m \in \mathbb{Z}} f_{m} \sigma(m)\left(g_{n-m}\right)\right) u_{n}
$$




\section{Introduction}

for $\sum_{n \in \mathbb{Z}} f_{n} u_{n}, \sum_{n \in \mathbb{Z}} g_{n} u_{n} \in C(X) \rtimes^{\sigma} \mathbb{Z}$. Note that if $X$ is compact and Hausdorff, so that $C^{*}(X, s)$ is defined, then the skew group algebra $C(X) \rtimes^{\sigma} \mathbb{Z}$ is a norm dense complex subalgebra of the $C^{*}$-algebra $C^{*}(X, s)$.

Theorem 2 (Svensson, Silvestrov and de Jeu [22]). Suppose that $s$ is a homeomorphism of a Tychonoff and Baire topological space $X$. The following assertions are equivalent:

(i) $(X, s)$ is topologically free;

(ii) if $I$ is a nonzero ideal of $C(X) \rtimes^{\sigma} \mathbb{Z}$, then $I \cap C(X) \neq\{0\}$;

(iii) $C(X)$ is a maximal abelian complex subalgebra of $C(X) \rtimes^{\sigma} \mathbb{Z}$.

In fact Svensson, Silvestrov and de Jeu loc. cit. prove a more general statement for skew group algebras $A \rtimes^{\sigma} \mathbb{Z}$ defined by complex subalgebras $A$ of $C(X)$ satisfying certain extra conditions, see Theorem 4.5 in [22] for more details. For related results by the same authors, see [20] and [21].

In the above discussion the dynamics is generated by a single continuous function. Therefore, all powers of this function commute which, in particular, means that we have an abelian action on the space. This raises the question if there is a nonabelian version of Theorem 2. Another natural question is if there is a version of the same theorem that holds for dynamics defined by families of partial functions on a space, that is functions that do not necessarily have the same domain or codomain. In this article we simultaneously address both of these questions by introducing category dynamical systems. These are defined by families, stable under composition, of continuous maps between potentially different topological spaces. We show generalizations of Theorem 2 to the case of skew category algebras defined by these maps and spaces (see Theorem 3 below and Theorem 20. Theorem 23 and Theorem 24 in Section 31). To be more precise, suppose that $G$ is a category. The family of objects of $G$ is denoted by ob $(G)$; we will often identify an object in $G$ with its associated identity morphism. The family of morphisms in $G$ is denoted by $\operatorname{mor}(G)$; by abuse of notation, we will often write $n \in G$ when we mean $n \in \operatorname{mor}(G)$. Throughout the article $G$ is assumed to be small, that is with the property that $\operatorname{mor}(G)$ is a set. The domain and codomain of a morphism $n$ in $G$ is denoted by $d(n)$ and $c(n)$ respectively. We let $G^{(2)}$ denote the collection of composable pairs of morphisms in $G$, that is all $(m, n)$ in $\operatorname{mor}(G) \times \operatorname{mor}(G)$ satisfying $d(m)=c(n)$. For each $e \in \operatorname{ob}(G)$, let $G_{e}$ denote the collection of $n \in \operatorname{mor}(G)$ with $d(n)=c(n)=e$. Let $G^{\text {op }}$ denote the opposite category of $G$. We let Top denote the category having topological spaces as objects and continuous functions as morphisms. Suppose that $s: G \rightarrow$ Top $^{\text {op }}$ is a (covariant) functor; in that case we say that $s$ is a category dynamical system. If $G$ is a groupoid, that is a category where all morphisms are isomorphisms, then we say that $s$ is a groupoid dynamical system. If $e \in \mathrm{ob}(G)$, then we say that an element $x \in s(e)$ is periodic if there is a nonidentity $n: e \rightarrow e$ in $G$ such that $s(n)(x)=x$; an element of $s(e)$ which is not periodic is called aperiodic. We say that $s$ is topologically free if for each $e \in \mathrm{ob}(G)$, the set of aperiodic elements of $s(e)$ is dense in $s(e)$. For each $e \in \operatorname{ob}(G)$, we let $C(e)$ denote the set of continuous complex valued functions on $s(e)$. For each $n \in G$ the functor $s$ induces a map $\sigma(n): C(d(n)) \rightarrow C(c(n))$ by the relation 


\section{Skew Category Algebras}

$\sigma(n)(f)=f \circ s(n)$, for $f \in C(d(n))$. If we use the terminology introduced in Section 2. then the map $\sigma$ defines a skew category system (see Definition 4). In this section, we show how one to each skew category system may associate a so called skew category algebra $A \rtimes^{\sigma} G$ (see also [15] for a more general construction) where $A=\oplus_{e \in \operatorname{ob}(G)} C(e)$. In Section 2, we also investigate the connection between maximal commutativity of $A$ in $A \rtimes^{\sigma} G$ and the way in which ideals intersect $A$ (see Proposition 12). In Section 3 , we apply these results to obtain connections between topological freeness of the category dynamical system $s$, ideal properties of $A \rtimes^{\sigma} G$ and maximal commutativity of $A$ in $A \rtimes^{\sigma} G$ (see Theorems 20, 23 and 24). In particular, in the end of the article, we show the following generalization of Theorem 2 from the additive group of integers to a large class of groupoids.

Theorem 3. Suppose that $s: G \rightarrow$ Top $^{\text {op }}$ is a groupoid dynamical system with the property that for each $e \in \mathrm{ob}(G)$, the group of all morphisms $e \rightarrow e$ is countable and $s(e)$ is Tychonoff and Baire. If we put $A=\oplus_{e \in \mathrm{ob}(G)} C(e)$, then the following assertions are equivalent:

(i) $s$ is topologically free;

(ii) if $I$ is a nonzero ideal of $A \rtimes^{\sigma} G$, then $I \cap A \neq\{0\}$;

(iii) the ring $A$ is a maximal abelian complex subalgebra of $A \rtimes^{\sigma} G$.

\section{Skew Category Algebras}

In this section, we define skew category systems $(A, G, \sigma)$ (see Definition 4) and skew category algebras $A \rtimes^{\sigma} G$ (see Definition 6). Thereafter, we investigate the existence of identity elements in skew category algebras (see Proposition 10). In the end of this section, we investigate the relation between maximal commutativity of $A$ and the ideal intersection property in skew category algebras $A \rtimes^{\sigma} G$ (see Proposition 11 and Proposition (12). These results will be applied to category dynamical systems in Section 3

Conventions. Let $R$ be an associative ring. The identity map $R \rightarrow R$ is denoted by $\operatorname{id}_{R}$. If $R$ is unital then the identity element of $R$ is nonzero and is denoted by $1_{R}$. The category of unital rings is denoted by Ring. We say that a subset $R^{\prime}$ of $R$ is a subring of $R$ if it is itself a ring under the binary operations of $R$. We always assume that ring homomorphisms between unital rings respect the identity elements. If $A$ is a subset of $R$, then the commutant of $A$ in $R$ is the set of elements of $R$ that commute with every element of $A$. If $A$ is a commutative subring of $R$, then $A$ is called maximal commutative in $R$ if the commutant of $A$ in $R$ equals $A$. All ideals of rings are supposed to be twosided. If $R$ is commutative and $x \in R$, then $\operatorname{Ann}(x)$ denotes the ideal of $R$ consisting of all $y \in R$ satisfying $x y=0$.

Definition 4. By a skew category system we mean a triple $(A, G, \sigma)$ where $G$ is a (small) category, $A$ is the direct sum of unital rings $A_{e}$, for $e \in \mathrm{ob}(G)$, and $\sigma$ is a functor $G \rightarrow$ Ring satisfying $\sigma(n): A_{d(n)} \rightarrow A_{c(n)}$, for $n \in G$. 


\section{Skew Category Algebras}

Remark 5. Suppose that $(A, G, \sigma)$ is a skew category system. The fact that $\sigma$ is a functor $G \rightarrow$ Ring can be formulated in terms of maps by saying that

$$
\sigma(e)=\operatorname{id}_{A_{e}},
$$

for all $e \in \mathrm{ob}(G)$, and

$$
\sigma(m) \sigma(n)=\sigma(m n)
$$

for all $(m, n) \in G^{(2)}$.

Definition 6. If $(A, G, \sigma)$ is a skew category system, then we let $A \rtimes^{\sigma} G$ denote the collection of formal sums $\sum_{n \in G} a_{n} u_{n}$, where $a_{n} \in A_{c(n)}, n \in G$, are chosen so that all but finitely many of them are nonzero. Define addition and multiplication on $A \rtimes^{\sigma} G$ by

$$
\left(\sum_{n \in G} a_{n} u_{n}\right)+\left(\sum_{n \in G} b_{n} u_{n}\right)=\sum_{n \in G}\left(a_{n}+b_{n}\right) u_{n}
$$

respectively

$$
\left(\sum_{n \in G} a_{n} u_{n}\right)\left(\sum_{n \in G} b_{n} u_{n}\right)=\sum_{n \in G}\left(\sum_{\substack{\left(m, m^{\prime}\right) \in G^{(2)} \\ m m^{\prime}=n}} a_{m} \sigma(m)\left(b_{m^{\prime}}\right)\right) u_{n}
$$

for $\sum_{n \in G} a_{n} u_{n}, \sum_{n \in G} b_{n} u_{n} \in A \rtimes^{\sigma} G$. It is clear that these operations define a (sometimes nonunital, see Proposition (10) ring structure on $A \rtimes^{\sigma} G$. We call $A \rtimes^{\sigma} G$ the skew category algebra defined by $(A, G, \sigma)$.

Remark 7. If $G$ is a groupoid, then (6) can be rewritten in the following slightly simpler form

$$
\left(\sum_{n \in G} a_{n} u_{n}\right)\left(\sum_{n \in G} b_{n} u_{n}\right)=\sum_{n \in G}\left(\sum_{\substack{m \in G ; \\ c(m)=c(n)}} a_{m} \sigma(m)\left(b_{m^{-1} n}\right)\right) u_{n} .
$$

If $G$ equals the additive group $\mathbb{Z}$, then (7) coincides with (2).

Remark 8. Suppose that $T:=A \rtimes^{\sigma} G$ is a skew category algebra. If we for each $n \in G$, put $T_{n}=A_{c(n)} u_{n}$, then $T=\oplus_{n \in G} T_{n}, T_{m} T_{n}=T_{m n}$, for $(m, n) \in G^{(2)}$, and $T_{m} T_{n}=\{0\}$, otherwise. In the language of [9], [10] and [16] this means that a skew category algebra is a strongly category graded ring.

Definition 9. Given a nonzero element $x=\sum_{n \in G} a_{n} u_{n}$ in the skew category algebra $A \rtimes^{\sigma} G$, we put

$$
\operatorname{Dom}(x)=\left\{d(n) \mid n \in G \text { and } a_{n} \neq 0\right\}
$$

and

$$
\operatorname{Cod}(x)=\left\{c(n) \mid n \in G \text { and } a_{n} \neq 0\right\}
$$




\section{Skew Category Algebras}

Proposition 10. Given a nonzero element $x$ in the skew category algebra $A \rtimes^{\sigma} G$, there are unique elements $l(x)$ and $r(x)$ in $A \rtimes^{\sigma} G$ subject to the condition that $l(x) y=y$ and $z r(x)=z$ for all elements $y$ and $z$ in $A \rtimes^{\sigma} G$ satisfying $\operatorname{Cod}(x)=\operatorname{Cod}(y)$ and $\operatorname{Dom}(x)=\operatorname{Dom}(z)$, namely $l(x)=\sum_{e \in \operatorname{Cod}(x)} 1_{A_{e}} u_{e}$ and $r(x)=\sum_{e \in \operatorname{Dom}(x)} 1_{A_{e}} u_{e}$. In particular, this implies that $A \rtimes^{\sigma} G$ is unital if and only if $\mathrm{ob}(G)$ is finite; in that case, the multiplicative identity of $A \rtimes^{\sigma} G$ is $\sum_{e \in \mathrm{ob}(G)} 1_{A_{e}} u_{e}$.

Proof. Suppose that $x=\sum_{n \in G} a_{n} u_{n}$ is an element of $A \rtimes^{\sigma} G$ satisfying $l(x) y=y$ and $z r(x)=z$ for all elements $y$ and $z$ in $A \rtimes^{\sigma} G$ with $\operatorname{Cod}(x)=\operatorname{Cod}(y)$ and $\operatorname{Dom}(x)=$ $\operatorname{Dom}(z)$. Suppose that $l(x)=\sum_{n \in G} b_{n} u_{n}$ for some $b_{n} \in A_{c(n)}$ satisfying $b_{n}=0$ for all but finitely many $n \in G$. If we put $y=\sum_{e \in \operatorname{Cod}(x)} 1_{A_{e}} u_{e}$, then $\operatorname{Cod}(x)=$ $\operatorname{Cod}(y)$. Hence, by the assumptions, we get that $\sum_{e \in \operatorname{Cod}(x)} 1_{A_{e}} u_{e}=y=l(x) y=$ $\left(\sum_{n \in G} b_{n} u_{n}\right)\left(\sum_{e \in \operatorname{Cod}(x)} 1_{A_{e}} u_{e}\right)=\sum_{e \in \operatorname{Cod}(x)} \sum_{n \in G, d(n)=e} b_{n} u_{n}$. This implies that $b_{n}=$ $1_{A_{c(n)}}$, for $n \in \operatorname{Cod}(x)$, and $b_{n}=0$, otherwise. This means that $l(x)=\sum_{e \in \operatorname{Cod}(x)} 1_{A_{e}} u_{e}$. The statement concerning $r(x)$ is treated similarly. On the other hand, it is clear that $l(x)$ and $r(x)$ as defined above satisfy the required property. The last part follows immediately from the above.

Proposition 11. If $A \rtimes^{\sigma} G$ is a skew category algebra with $A$ commutative, then

(a) the commutant of $A$ in $A \rtimes^{\sigma} G$ is the collection of elements of $A \rtimes^{\sigma} G$ of the form $\sum_{e \in \mathrm{ob}(G)} \sum_{n \in G_{e}} a_{n} u_{n}$ satisfying $\sigma(n)(a)-a \in \operatorname{Ann}\left(a_{n}\right)$, for all $e \in \mathrm{ob}(G)$, all $n \in G_{e}$ and all $a \in A_{e}$;

(b) the subring $A$ is maximal commutative in $A \rtimes^{\sigma} G$ if and only if there for all $e \in \mathrm{ob}(G)$, all nonidentity $n \in G_{e}$ and all nonzero $a_{n} \in A_{e}$, is a nonzero $a \in A_{e}$ with the property that $\sigma(n)(a)-a \notin \operatorname{Ann}\left(a_{n}\right)$;

(c) if every $A_{e}$, for $e \in \mathrm{ob}(G)$, is an integral domain, then $A$ is maximal commutative in $A \rtimes^{\sigma} G$ if and only if there for every $e \in \mathrm{ob}(G)$ and every nonidentity $n \in G_{e}$ is $a \in A_{e}$ with the property that $\sigma(n)(a) \neq a$.

Proof. Let the commutant of $A$ in $A \rtimes^{\sigma} G$ be denoted by $A^{\prime}$.

(a) Take $x=\sum_{n \in G} a_{n} u_{n}$ in $A^{\prime}$. From the fact that the equality $1_{A_{e}} u_{e} x=x 1_{A_{e}} u_{e}$ holds for all $e \in \operatorname{ob}(G)$, it follows that $a_{n}=0$ whenever $d(n) \neq c(n)$. Take $e \in \operatorname{ob}(G)$ and $a \in A_{e}$. From the fact that the equality $a u_{e} x=x a u_{e}$ holds it follows that if $n \in G_{e}$, then $a a_{n}=\sigma(n)(a) a_{n}$, that is that $a-\sigma(n)(a) \in \operatorname{Ann}\left(a_{n}\right)$. On the other hand, it is clear that elements of $A \rtimes_{\alpha}^{\sigma} G$ of the form $\sum_{e \in \mathrm{ob}(G)} \sum_{n \in G_{e}} a_{n} u_{n}$ satisfying $\sigma(n)(a)-a \in \operatorname{Ann}\left(a_{n}\right)$, for $e \in \operatorname{ob}(G), n \in G_{e}$ and $a \in A_{e}$, belongs to $A^{\prime}$.

(b) follows from (a) and (c) follows from (b).

Proposition 12. Suppose that $A \rtimes^{\sigma} G$ is a skew category algebra with $A$ commutative. Consider the following assertions:

(i) if $I$ is a nonzero ideal of $A \rtimes^{\sigma} G$, then $I \cap A \neq\{0\}$;

(ii) the subring $A$ is maximal commutative in $A \rtimes^{\sigma} G$. 


\section{Skew Category Algebras}

Then:

(a) (i) implies (ii);

(b) (ii) does not imply (i) for all categories $G$;

(c) if $G$ is a groupoid, then (i) holds if and only if (ii) holds.

Proof. (a) We show the contrapositive statement. Suppose that $A$ is not maximal commutative in $A \rtimes^{\sigma} G$. Then, by Proposition 11(b), there exists some $e \in \mathrm{ob}(G)$, some nonidentity $n \in G_{e}$ and some nonzero $a_{n} \in A_{e}$ with the property that $\sigma(n)(a)-a \in \operatorname{Ann}\left(a_{n}\right)$ for all $a \in A_{e}$, that is such that $a_{n} \sigma(n)(a)=a_{n} a$ for all $a \in A_{e}$. Let $I$ be the nonzero ideal of $A \rtimes^{\sigma} G$ generated by the element $a_{n} u_{e}-a_{n} u_{n}$ and define the homomorphism of abelian groups $\varphi: A \rtimes^{\sigma} G \rightarrow A$ by the additive extension of the relation $\varphi\left(x u_{t}\right)=x$, for $t \in G$ and $x \in A_{c(t)}$. We claim that $I \subseteq \operatorname{ker}(\varphi)$. If we assume that the claim holds, then, since $\left.\varphi\right|_{A}=\mathrm{id}_{A}$, it follows that $A \cap I=\left.\varphi\right|_{A}(A \cap I) \subseteq \varphi(I)=\{0\}$. Now we show the claim. By the definition of $I$ it follows that it is enough to show that $\varphi$ maps elements of the form $x u_{r}\left(a_{n} u_{e}-a_{n} u_{n}\right) y u_{t}$ to zero, where $x \in A_{c(r)}, y \in A_{c(t)}$ and $r, t \in G$ satisfy $d(r)=e=c(t)$. However, since $a_{n} \sigma(n)(a)=a_{n} a$ for all $a \in A_{e}$, we get that $x u_{r}\left(a_{n} u_{e}-a_{n} u_{n}\right) y u_{t}=x u_{r}\left(a_{n} y u_{t}-a_{n} y u_{n} u_{t}\right)=x u_{r}\left(a_{n} y u_{t}-a_{n} y u_{n t}\right)=$ $x \sigma(r)\left(a_{n} y\right) u_{r t}-x \sigma(r)\left(a_{n} y\right) u_{r n t}$ which, obviously, is mapped to zero by $\varphi$.

(b) We will show that there even are monoids for which (ii) does not imply (i). Let $\mathbb{N}$ denote the nonnegative integers equipped with addition as operation and zero as a neutral element. Let $A$ denote the polynomial ring $\mathbb{C}[X]$ and fix a complex number $z$ which is not a root of unity, that is such that $z^{n} \neq 1$ for all positive integers $n$. For each $n \in \mathbb{N}$ define $\sigma(n): A \rightarrow A$ by $\sigma(n)(p(X))=p\left(z^{n} X\right)$, for $p(X) \in A$. It is clear that each $\sigma(n)$, for $n \in \mathbb{N}$, is a ring endomorphism of $A$. It is easy to check that if $p(X)$ is any nonconstant polynomial and $n$ is a positive integer, then $\sigma(n)(p(X)) \neq p(X)$. Since $A$ is an integral domain, this implies, by Proposition 11(c), that $A$ is maximal commutative in $A \rtimes^{\sigma} G$. However, if we let $I$ denote the ideal generated by $u_{1}$, then it is clear that $A \cap I=\{0\}$.

(c) By (a) it follows that (i) implies (ii). Now we show the contrapositive statement of (ii) implies (i). Let $C$ denote the commutant of $A$ in $A \rtimes^{\sigma} G$ and suppose that $I$ is a twosided ideal of $A \rtimes^{\sigma} G$ with the property that $I \cap C=\{0\}$. We wish to show that $I=\{0\}$. Take $x \in I$. If $x \in C$, then by the assumption $x=0$. Therefore we now assume that $x=\sum_{s \in G} a_{s} u_{s} \in I, a_{s} \in A_{c(s)}, s \in G$, and that $x$ is chosen so that $x \notin C$ with the set $S:=\left\{s \in G \mid a_{s} \neq 0\right\}$ of least possible cardinality $N$. Seeking a contradiction, suppose that $N$ is positive. First note that there is $e \in c(x)$ with $u_{e} x \in I \backslash C$. In fact, if $u_{e} x \in C$ for all $e \in c(x)$, then $x=l(x) x=\sum_{e \in c(x)} u_{e} x \in C$ which is a contradiction. By minimality of $N$ we can assume that $c(s)=e, s \in S$, for some fixed $e \in \operatorname{ob}(G)$. Take $t \in S$ and consider the nonzero element $x^{\prime}:=x u_{t^{-1}} \in I$. Since $I \cap C=\{0\}$, we get that $x^{\prime} \in I \backslash C$. Take $a=\sum_{f \in \mathrm{ob}(G)} b_{f} u_{f} \in A$. Then $I \ni x^{\prime \prime}:=a x^{\prime}-x^{\prime} a=\sum_{s \in S} a_{s}\left(b_{d(s)}-\sigma_{s}\left(b_{e}\right)\right) u_{s}$. Since the summand for $s=e$ vanishes, we get, by the assumption on $N$, that $x^{\prime \prime}=0$. Since $a \in A$ was arbitrarily chosen, we get that $x^{\prime} \in C$ which is a contradiction. Therefore $N=0$ and hence $S=\emptyset$ which in turn implies that $x=0$. Since $x \in I$ was arbitrarily chosen, we finally get that $I=\{0\}$. 


\section{Category Dynamical Systems}

Remark 13. For other results related to the implication (i) implies (ii) in Proposition 12. see [15]. The implication (ii) implies (i) in Proposition 12 actually holds for all nondegenerate groupoid graded rings, see [16].

\section{Category Dynamical Systems}

In this section, we show generalizations of Theorem 2 to the case of skew category algebras defined by category topological dynamical systems (see Theorem 20. Theorem 23 and Theorem 24). We use these results to prove Theorem 3, To this end, we apply results from the previous section to show results about the commutant of $A$ in the corresponding skew category algebra $A \rtimes^{\sigma} G$ (see Proposition [18). In the end of this section, we discuss the implications of these results for the connection between dynamical systems defined by partially defined functions (see Definition 26) and properties of the corresponding skew category algebras (see Example 28).

Conventions. Let $s: G \rightarrow$ Top $^{\text {op }}$ be a category dynamical system and suppose that $R$ denotes a commutative unital topological ring, that is a commutative unital ring which is also a topological space such that both the addition and the multiplication are continuous as maps $R \times R \rightarrow R$, where $R \times R$ carries the product topology. Take $e \in \mathrm{ob}(G)$. We let $C(s(e), R)$ denote the $R$-algebra of continuous maps $s(e) \rightarrow R$; we let $1_{e}$ denote the map $s(e) \rightarrow R$ that sends each $x \in s(e)$ to $1_{R}$; if $R$ equals the complex numbers, then we let $C(s(e), R)$ be denoted by $C(e)$. For each $n \in G$, we let $\sigma(n)$ denote the function $C(s(d(n)), R) \rightarrow C(s(c(n)), R)$ defined by $\sigma(n)(f)=f \circ s(n)$, for $f \in C(s(d(n)), R)$. For the rest of the article, we suppose that

(i) $A_{e}$ denotes a subring of $C(s(e), R)$;

(ii) if $n \in G$ and $f \in A_{d(n)}$, then $\sigma(n)(f)$ belongs to $A_{c(n)}$;

(iii) $A$ denotes the direct $\operatorname{sum} \oplus_{e \in \mathrm{ob}(G)} A_{e}$.

The support of $f \in C(s(e), R)$ is denoted by $\operatorname{Supp}(f)$ and is the set of $x \in s(e)$ such that $f(x) \neq 0$. Furthermore, we say that a nonempty subset of $s(e)$ is a domain of uniqueness for $A_{e}$ if every function of $A_{e}$ that vanishes on it, vanishes on the whole of $s(e)$. We also say that the ring $A_{e}$ separates elements of $s(e)$ if for each pair of distinct elements $x$ and $y$ in $s(e)$ there exists a function $f$ in $A_{e}$ such that $f(x) \neq f(y)$.

Proposition 14. The triple $(A, G, \sigma)$ is a skew category system.

Proof. We need to check conditions (3) and (4) from Remark 5. Take $e \in \operatorname{ob}(G)$ and $f \in A_{e}$. Then $\sigma(e)(f)=f \circ s(e)=f$. Therefore $\sigma(e)=\operatorname{id}_{A_{e}}$. Take $(m, n) \in G^{(2)}$ and $f \in A_{d(n)}$. Then $\sigma(m) \sigma(n)(f)=\sigma(m)(f \circ s(n))=f \circ s(n) \circ s(m)=f \circ(s(m) \circ$ op $s(n))=$ $f \circ s(m n)=\sigma(m n)(f)$. Therefore $\sigma(m) \sigma(n)=\sigma(m n)$.

Definition 15. If $e \in \mathrm{ob}(G), x \in s(e)$ and $n \in G_{e}$, then we put

(i) $\operatorname{Sep}_{A_{e}}^{n}=\left\{x \in s(e) \mid\right.$ there is $f \in A_{e}$ with $\left.f(x) \neq \sigma(n)(f)(x)\right\}$; 


\section{Category Dynamical Systems}

(ii) $\operatorname{Per}_{A_{e}}^{n}=\left\{x \in s(e) \mid\right.$ for all $f \in A_{e}, f(x)=\sigma(n)(f)(x)$ holds $\}$;

(iii) $\operatorname{Sep}_{e}^{n}=\{x \in s(e) \mid x \neq s(n)(x)\}$;

(iv) $\operatorname{Per}_{e}^{n}=\{x \in s(e) \mid x=s(n)(x)\}$;

(v) $\operatorname{Per}_{A_{e}}^{\infty}=\bigcap_{n \in G_{e} \backslash\{e\}} \operatorname{Sep}_{A_{e}}^{n}$;

(vi) $\operatorname{Per}_{e}^{\infty}=\bigcap_{n \in G_{e} \backslash\{e\}} \operatorname{Sep}_{e}^{n}$;

(vii) $\operatorname{Orb}_{e}(x)=\left\{s(n)(x) \mid n \in G_{e}\right\}$.

Proposition 16. If $e \in \mathrm{ob}(G)$ and $n \in G_{e}$, then

(a) $s(e) \backslash \operatorname{Sep}_{A_{e}}^{n}=\operatorname{Per}_{A_{e}}^{n}$;

(b) $s(e) \backslash \operatorname{Sep}_{e}^{n}=\operatorname{Per}_{e}^{n}$;

(c) $s(e) \backslash \operatorname{Per}_{A_{e}}^{n}=\operatorname{Sep}_{A_{e}}^{n}$;

(d) $s(e) \backslash \operatorname{Per}_{e}^{n}=\operatorname{Sep}_{e}^{n}$;

(e) $\operatorname{Per}_{e}^{n} \subseteq \operatorname{Per}_{A_{e}}^{n}$;

(f) $\operatorname{Sep}_{A_{e}}^{n} \subseteq \operatorname{Sep}_{e}^{n}$;

(g) $\operatorname{Per}_{e}^{\infty} \subseteq \operatorname{Sep}_{e}^{n}$.

Proof. (a), (b) and (e) are trivial. (c), (d) and (f) follow from (a), (b) and (e) by taking complements. (g) follows from the definition of $\mathrm{Per}_{e}^{\infty}$.

Proposition 17. If $e \in \mathrm{ob}(G)$ and $n \in G_{e}$, then

(a) if $A_{e}$ separates the elements of $s(e)$, then $\operatorname{Per}_{e}^{n}=\operatorname{Per}_{A_{e}}^{n}$ and $\operatorname{Sep}_{e}^{n}=\operatorname{Sep}_{A_{e}}^{n}$;

(b) if $s(e)$ is a Hausdorff topological space and $s(n)$ is continuous, then $\operatorname{Per}_{e}^{n}$ is closed and $\operatorname{Sep}_{e}^{n}$ is open;

(c) if $s(e)$ is a topological space and $A_{e}$ is an $R$-subalgebra of $C(s(e), R)$ with the property that each set $f^{-1}(\{0\})$, for $f \in A_{e}$, is closed, then $\operatorname{Per}_{A_{e}}^{n}$ is closed and $\operatorname{Sep}_{A_{e}}^{n}$ is open.

Proof. (a) The inclusion $\operatorname{Per}_{e}^{n} \subseteq \operatorname{Per}_{A_{e}}^{n}$ follows from Proposition (16)(e). The inclusion $\operatorname{Per}_{e}^{n} \supseteq \operatorname{Per}_{A_{e}}^{n}$ follows from the fact that $A_{e}$ separates the elements of $s(e)$. The equality $\operatorname{Sep}_{e}^{n}=\operatorname{Sep}_{A_{e}}^{n}$ follows from the equality $\operatorname{Per}_{e}^{n}=\operatorname{Per}_{A_{e}}^{n}$ and Proposition 16(c)(d).

(b) It is well known that the set of fixed points of a continuous function on a Hausdorff topological space is a closed set. Therefore, $\mathrm{Per}_{e}^{n}$ is closed. By Proposition 16(b) it follows that $\operatorname{Sep}_{e}^{n}$ is open.

(c) Take $f \in A_{e}$. By the assumptions it follows that the set

$$
\{x \in s(e) \mid f(x)=\sigma(n)(f)(x)\}=(f-\sigma(n)(f))^{-1}(\{0\})
$$


is closed. Therefore the set

$$
\operatorname{Per}_{A_{e}}^{n}=\bigcap_{f \in A_{e}}(f-\sigma(n)(f))^{-1}(\{0\})
$$

is closed. The last part follows from Proposition 16(c).

Proposition 18. Suppose that $R$ is an integral domain.

(a) The commutant of $A$ in $A \rtimes^{\sigma} G$ is the set of elements of $A \rtimes^{\sigma} G$ of the form $\sum_{e \in \mathrm{ob}(G)} \sum_{n \in G_{e}} f_{n} u_{n}$ satisfying $\operatorname{Supp}\left(f_{n}\right) \subseteq \operatorname{Per}_{A_{e}}^{n}$, for $e \in \mathrm{ob}(G)$ and $n \in G_{e}$. In particular, $A$ is maximal commutative in $A \rtimes^{\sigma} G$ if and only if for each $e \in \mathrm{ob}(G)$ and each nonidentity $n \in G_{e}$, the set $\operatorname{Sep}_{A_{e}}^{n}$ is a domain of uniqueness for $A_{e}$.

(b) If for each $e \in \mathrm{ob}(G)$, Ae separates the elements of $s(e)$, then the commutant of $A$ in $A \rtimes^{\sigma} G$ is the set of elements of $A \rtimes^{\sigma} G$ of the form $\sum_{e \in \mathrm{ob}(G)} \sum_{n \in G_{e}} f_{n} u_{n}$ satisfying $\operatorname{Supp}\left(f_{n}\right) \subseteq \operatorname{Per}_{e}^{n}$, for $e \in \mathrm{ob}(G)$ and $n \in G_{e}$. In particular, $A$ is maximal commutative in $A \rtimes^{\sigma} G$ if and only if for each $e \in \mathrm{ob}(G)$ and each nonidentity $n \in G_{e}$, the set $\mathrm{Sep}_{e}^{n}$ is a domain of uniqueness for $A_{e}$.

Proof. (a) By Proposition 11(a) we get that the commutant of $A$ in $A \rtimes^{\sigma} G$ is the set of elements $\sum_{e \in \mathrm{ob}(G)} \sum_{n \in G_{e}} f_{n} u_{n}$ in $A \rtimes^{\sigma} G$ satisfying $\sigma(n)(g)-g \in \operatorname{Ann}\left(f_{n}\right)$ for all $g \in A_{e}$. This means that $f_{n}(\sigma(n)(g)-g)=0$ for all $g \in A_{e}$. From the fact that $R$ is an integral domain it follows that if $x \in \operatorname{Supp}\left(f_{n}\right)$, then $\sigma(n)(g)(x)-g(x)=0$, for all $g \in A_{e}$, that is $x \in \operatorname{Per}_{A_{e}}^{n}$. Therefore we get that $\operatorname{Supp}\left(f_{n}\right) \subseteq \operatorname{Per}_{A_{e}}^{n}$, for $e \in \operatorname{ob}(G)$ and $n \in G_{e}$. By Proposition [11(b) it follows that $A$ is maximal commutative in $A \rtimes^{\sigma} G$ if and only if for each choice of $e \in \mathrm{ob}(G)$ and nonidentity $n \in G_{e}$, we get that if $f_{n} \in A_{e}$ is nonzero there exists $g \in A_{e}$ with $\sigma(n)(g)-g \notin \operatorname{Ann}\left(f_{n}\right)$, that is such that $f_{n}(\sigma(n)(g)-g) \neq 0$. This is in turn equivalent to the contrapositive statement that to each choice of $e \in \operatorname{ob}(G)$ and nonidentity $n \in G_{e}$, the equality $\left.f_{n}\right|_{\operatorname{Sep}_{A_{e}}^{n}}=0$ implies that $f_{n}=0$, that is that $\operatorname{Sep}_{A_{e}}^{n}$ is a domain of uniqueness for $A_{e}$.

(b) This follows immediately from (a) and Proposition 17(a).

Remark 19. Proposition 18 generalizes Theorem 3.3 and Theorem 3.5 in [20].

Theorem 20. Consider the following three assertions:

(i) $s$ is topologically free;

(ii) if $I$ is a nonzero ideal of $A \rtimes^{\sigma} G$, then $I \cap A \neq\{0\}$;

(iii) $A$ is maximal commutative in $A \rtimes^{\sigma} G$.

Then:

(a) (ii) implies (iii);

(b) if $G$ is a groupoid, then (ii) holds if and only if (iii) holds; 


\section{Category Dynamical Systems}

(c) if $R$ is an integral domain and for each $e \in \mathrm{ob}(G)$ the function space $A_{e}$ separates elements of $s(e)$ and for each $f \in A_{e}$, the set $f^{-1}(\{0\})$ is closed in $s(e)$, then (i) implies (iii); this holds e.g. if $\{0\}$ is closed in $R$, which, in turn, holds e.g. when $R$ is Hausdorff;

(d) if $R$ is an integral domain and for each $e \in \mathrm{ob}(G)$, the space $s(e)$ is Hausdorff and Baire, the function space $A_{e}$ separates elements of $s(e)$ and has the property that for each nonempty open subset $U$ of $s(e)$, there is a nonzero $f \in A_{e}$ that vanishes on $s(e) \backslash U$, then (iii) implies (i).

Proof. (a) and (b) follow immediately from Proposition 12(a)(c).

(c) Take an $e \in \operatorname{ob}(G)$ and a nonidentity $n \in G_{e}$. Suppose that the set $\operatorname{Per}_{e}^{\infty}$ is dense in $s(e)$. By Proposition 16(g), the inclusion $\operatorname{Per}_{e}^{\infty} \subseteq \operatorname{Sep}_{e}^{n}$ holds. By Proposition 17(a) it follows that $\operatorname{Sep}_{A_{e}}^{n}$ is dense in $s(e)$. If $f \in A_{e}$ satisfies $f(x)=0$, for $x \in \operatorname{Sep}_{A_{e}}^{n}$, then $f^{-1}(\{0\})$ is a closed subset of $s(e)$ containing the dense subset $\operatorname{Sep}_{A_{e}}^{n}$ of $s(e)$. Therefore, $f^{-1}(\{0\})=s(e)$ and hence $\operatorname{Sep}_{A_{e}}^{n}$ is a domain of uniqueness for $A_{e}$. By Proposition 18(a) it follows that $A$ is maximal commutative in $A \rtimes^{\sigma} G$.

(d) We show the contrapositive of (iii) implies (i). Suppose that for some $e \in \operatorname{ob}(G)$, the set $\operatorname{Per}_{e}^{\infty}$ is not dense in $s(e)$. Since $s(e)$ is Hausdorff, it follows, by Proposition 17(b), that all the sets $\operatorname{Sep}_{e}^{n}$, for nonidentity $n \in G_{e}$, are open. Therefore, since $s(e)$ is Baire, there is some nonidentity $n \in G_{e}$ for which $\operatorname{Sep}_{e}^{n}$ is not dense, that is such that $\operatorname{Per}_{e}^{n}$ has a nonempty interior $U$. By the assumption on $A_{e}$ there is a nonzero $f \in A_{e}$ that vanishes outside $U$. Hence, by Proposition 18(b), it follows that $f u_{n}$ belongs to the commutant of $A$ in $A \rtimes^{\sigma} G$. But since obviously $f u_{n}$ does not belong to $A$, the subring $A$ is not maximal commutative in $A \rtimes^{\sigma} G$.

Remark 21. Theorem 20 generalizes Theorem 3.7 in [20].

Remark 22. If $X$ is a Hausdorff and locally compact topological space, then $X$ is Baire and all the complex algebras

- $C(X)$

- $C_{c}(X)=\{f \in C(X) \mid \operatorname{Supp}(f)$ compact $\}$

- $C_{b}(X)=\{f \in C(X) \mid f$ bounded $\}$

- $C_{0}(X)=\{f \in C(X) \mid$ for every $\epsilon>0,\{x|| f(x) \mid \geq \epsilon\}$ is compact $\}$

separate elements and have the property that to each nonempty open subset $U$ of $X$, there is a nonzero function $f$ in the algebra that vanishes on $X \backslash U$ (see e.g. [11]).

Theorem 23. Suppose that $s: G \rightarrow$ Top $^{\text {op }}$ is a groupoid dynamical system. If for each $e \in \operatorname{ob}(G)$ the topological space $s(e)$ is Hausdorff and locally compact and $A_{e}$ equals $C(e)$ (or $C_{c}(e)$; or $C_{b}(e)$; or $C_{0}(e)$ ), then the following three assertions are equivalent:

(i) $s$ is topologically free; 
(ii) if $I$ is a nonzero ideal of $A \rtimes^{\sigma} G$, then $I \cap A \neq\{0\}$;

(iii) the ring $A$ is a maximal abelian complex subalgebra of $A \rtimes^{\sigma} G$.

Proof. This follows from Theorem 20 and Remark 22

Theorem 24. Suppose that for each $e \in \mathrm{ob}(G)$ the sets $s(e)$ and $R$ carry the discrete topologies and $A_{e}$ equals the set of all functions $s(e) \rightarrow R$. Consider the following three assertions:

(i) $s$ is topologically free;

(ii) if $I$ is a nonzero ideal of $A \rtimes^{\sigma} G$, then $I \cap A \neq\{0\}$;

(iii) $A$ is maximal commutative in $A \rtimes^{\sigma} G$.

\section{Then:}

(a) (ii) implies (iii);

(b) if $G$ is a groupoid, then (ii) holds if and only if (iii) holds;

(c) if $R$ is an integral domain, then (i) holds if and only if (iii) holds;

(d) if there is $e \in \mathrm{ob}(G)$ and $x \in s(e)$ such that $G_{e}$ has cardinality greater than the cardinality of $\operatorname{Orb}_{e}(x)$ and $G_{e}$ is a divisible monoid, that is such that for any distinct $m, n \in G_{e}$, there is $p \in G_{e}$ with $m=n p$ or $n=m p$, then (iii) does not hold;

(e) if there is $e \in \mathrm{ob}(G)$ and $x \in s(e)$ such that $\operatorname{Orb}_{e}(x)$ is finite and $G_{e}$ is an infinite and divisible monoid, e.g. $(\mathbb{Z},+)$ or $(\mathbb{N},+)$, then (iii) does not hold.

Proof. (a), (b), and (c) follow immediately from Theorem 20.

(d) Suppose that $e \in \mathrm{ob}(G)$ and $x \in s(e)$ are chosen so that $G_{e}$ is divisible and has cardinality greater than the cardinality of $\operatorname{Orb}_{e}(x)$. Then there exist distinct $m, n \in G_{e}$ with $s(m)(x)=s(n)(x)$. Since $G_{e}$ is divisible, there is a nonidentity $p \in G_{e}$ with $m=n p($ or $n=m p)$. This implies that $s(n p)(x)=s(n)(x)$ (or $s(m p)(x)=s(m)(x)$ ), that is $s(p) s(n)(x)=s(n)(x)$ (or $s(p) s(m)(x)=s(m)(x)$ ). Hence $s(n)(x) \in \operatorname{Per}_{e}^{p}$ (or $\left.s(m)(x) \in \operatorname{Per}_{e}^{p}\right)$ which implies that $\operatorname{Per}_{e}^{p}$ is nonempty. The claim now follows from Theorem 20(d).

(e) follows from (d).

Definition 25. Recall that a topological space $X$ is called completely regular if given any open subset $U$ of $X$ and any $x \in U$, there is $f \in C(X)$ with $f(x)=1$ and $f(y)=0$, for $y \in X \backslash U$. Moreover, a topological space $X$ is called Tychonoff if it is completely regular and Hausdorff.

Proof of Theorem 3. This follows from Theorem 23 and Definition 25.

Definition 26. Suppose that $X$ is a topological space. By a partially defined dynamical system on $X$ we mean a collection $P$ of functions such that 


\section{Category Dynamical Systems}

- if $f \in P$, then the domain $d(f)$ of $f$ and the codomain $c(f)$ of $f$ are subsets of $X$ and $f$ is continuous as a function $d(f) \rightarrow c(f)$ where $d(f)$ and $c(f)$ are equipped with the relative topologies induced by the topology on $X$;

- if $f \in P$, then $\operatorname{id}_{d(f)} \in P$ and $\operatorname{id}_{c(f)} \in P$;

- if $f, g \in P$ are such that $d(f)=c(g)$, then $f \circ g \in P$.

We say that an element $x$ of $X$ is periodic with respect to $P$ if there is a nonidentity function $f$ in $P$ with $d(f)=c(f)$ and $f(x)=x$. An element $x$ is aperiodic with respect to $P$ if it is not periodic. We say that $P$ is topologically free if the set of aperiodic elements of $X$ is dense in $X$. By abuse of notation, we let $P$ denote the category having the domains and codomains of functions in $P$ as objects and the functions of $P$ as morphisms. We let the obvious functor $P \rightarrow$ Top be denoted by $t_{P}$. Let $G_{P}$ denote the opposite category of $P$ and let $s_{P}: G_{P} \rightarrow$ Top $^{\text {op }}$ denote the opposite functor of $t_{P}$. We will call $s_{P}$ the category dynamical system on $X$ defined by the partially defined dynamical system $P$.

Proposition 27. If $X$ is a topological space and $P$ is a partially defined dynamical system on $X$, then $P$ is topologically free if and only if $s_{P}$ is topologically free as a category dynamical system.

Proof. This follows immediately from the definition of topological freeness of $P$ and $s_{P}$.

To illustrate the above definitions and results, we end the article with a simple example of a partially defined dynamical system.

Example 28. Suppose that we let $X$ denote the real numbers equipped with its usual topology and we let $Y$ denote the set of nonnegative real numbers equipped with the relative topology induced by the topology on $X$. Let sqr : $X \rightarrow Y$ and sqrt : $Y \rightarrow X$ denote the square function and the square root function, respectively. Furthermore, let abs : $X \rightarrow X$ denote the absolute value. Let $P$ be the partially defined dynamical system with $\operatorname{ob}(P)=\{X, Y\}$ and $\operatorname{mor}(P)=\left\{\operatorname{id}_{X}, \operatorname{id}_{Y}\right.$, sqr, sqrt, abs $\}$. Then we get the following table of partial composition for $P$

\begin{tabular}{c|ccccc}
$\circ$ & $\operatorname{id}_{X}$ & $\operatorname{id}_{Y}$ & sqr & sqrt & abs \\
\hline $\operatorname{id}_{X}$ & $\operatorname{id}_{X}$ & $*$ & $*$ & sqrt & abs \\
$\operatorname{id}_{Y}$ & $*$ & $\operatorname{id}_{Y}$ & sqr & $*$ & $*$ \\
sqr & sqr & $*$ & $*$ & $\operatorname{id}_{Y}$ & sqr \\
sqrt & $*$ & sqrt & abs & $*$ & $*$ \\
abs & abs & $*$ & $*$ & sqrt & abs
\end{tabular}

Let $G=P^{\text {op }}$ and put $A_{X}=C(X, \mathbb{R})$ and $A_{Y}=C(Y, \mathbb{R})$. Take $f_{X}, f_{X}^{\prime}, g_{X}, g_{X}^{\prime}, h_{X}, h_{X}^{\prime} \in$ $A_{X}$ and $f_{Y}, f_{Y}^{\prime}, g_{Y}, g_{Y}^{\prime} \in A_{Y}$. Then the product of

$$
B_{1}:=f_{X} u_{\mathrm{id}_{X}}+g_{X} u_{\mathrm{abs}}+h_{X} u_{\mathrm{sqr}}+f_{Y} u_{\mathrm{id}_{Y}}+g_{Y} u_{\mathrm{sqrt}}
$$


and

$$
B_{2}:=f_{X}^{\prime} u_{\mathrm{id}_{X}}+g_{X}^{\prime} u_{\mathrm{abs}}+h_{X}^{\prime} u_{\mathrm{sqr}}+f_{Y}^{\prime} u_{\mathrm{id}_{Y}}+g_{Y}^{\prime} u_{\mathrm{sqrt}}
$$

in the skew category algebra $A \rtimes^{\sigma} G$ equals

$$
\begin{gathered}
B_{1} B_{2}=f_{X} f_{X}^{\prime} u_{\mathrm{id}_{X}}+\left(f_{X} g_{X}^{\prime}+g_{X}\left(f_{X}^{\prime} \circ \mathrm{abs}\right)+g_{X}\left(g_{X}^{\prime} \circ \mathrm{abs}\right)+h_{X}\left(g_{Y}^{\prime} \circ \mathrm{sqr}\right)\right) u_{\mathrm{abs}}+ \\
+\left(f_{X} h_{X}^{\prime}+h_{X}\left(f_{Y}^{\prime} \circ \mathrm{sqr}\right)+g_{X}\left(h_{X}^{\prime} \circ \mathrm{abs}\right)\right) u_{\mathrm{sqr}}+\left(f_{Y} f_{Y}^{\prime}+g_{Y}\left(h_{X}^{\prime} \circ \mathrm{sqrt}\right)\right) u_{\mathrm{id}_{Y}}+ \\
+\left(f_{Y} g_{Y}^{\prime}+g_{Y}\left(f_{X}^{\prime} \circ \text { sqrt }\right)+g_{Y}\left(g_{X}^{\prime} \circ \text { sqrt }\right)\right) u_{\mathrm{sqrt}} .
\end{gathered}
$$

Now we examine the properties (i), (ii) and (iii) in Theorem 23, It turns out that they are all false for our particular example.

Property (i) is false. In fact, the subset of periodic elements of $X$ with respect to $P$ is $Y$. Therefore, the set of aperiodic elements of $X$ is not dense in $\mathrm{X}$.

Property (ii) is false. Indeed, the ideal $I$ of $A \rtimes^{\sigma} G$ generated by $u_{\text {abs }}$ equals

$$
A_{X} u_{\mathrm{abs}}+A_{X} u_{\mathrm{sqr}}+A_{Y} u_{\mathrm{sqrt}}
$$

and hence has the property that $I \cap A=\{0\}$.

Property (iii) is false. By Proposition [18(b) it follows that the commutant of $A$ in $A \rtimes^{\sigma} G$ equals the set of elements of $A \rtimes^{\sigma} G$ of the form

$$
f_{X} u_{\mathrm{id}_{X}}+g_{X} u_{\mathrm{abs}}+f_{Y} u_{\mathrm{id}_{Y}}
$$

with $f_{X}, g_{X} \in A_{X}$ and $f_{Y} \in A_{Y}$ satisfying $\operatorname{Supp}\left(g_{X}\right) \subseteq Y$. Therefore $A$ is not maximal commutative in $A \rtimes^{\sigma} G$.

\section{Acknowledgements}

The second author was partially supported by The Swedish Research Council (postdoctoral fellowship no. 2010-918), The Danish National Research Foundation (DNRF) through the Centre for Symmetry and Deformation, The Crafoord Foundation, The Royal Physiographic Society in Lund, The Swedish Foundation for International Cooperation in Research and Higher Education (STINT) and The Swedish Royal Academy of Sciences.

\section{References}

[1] R. J. Archbold and J. S. Spielberg, Topologically free actions and ideals in discrete $C^{*}$-dynamical systems, Proc. of Edinburgh Math. Soc. 37 (1993), 119-124.

[2] B. Blackadar, Operator Algebras: Theory of $C^{*}$-algebras and von Neumann algebras, Encyclopaedia of Mathematical Sciences, 122. Operator Algebras and Noncommutative Geometry, III. Springer-Verlag, Berlin, 2006.

[3] E. G. Effros and F. Hahn, Locally compact transformation groups and $C^{*}$-algebras, Memoirs of the American Mathematical Society, no. 75, American Mathematical Society, Providence RI, 1967. 


\section{References}

[4] G. A. Elliott, Some simple $C^{*}$-algebras constructed as crossed products with discrete outer automorphism groups, Publ. Res. Inst. Math. Sci. 16 (1980), 299-311.

[5] S. Kawamura and J. Tomiyama, Properties of topological dynamical systems and corresponding $C^{*}$-algebras, Tokyo. J. Math. 13 (1990), 251-257.

[6] A. Kishimoto, Outer automorphisms and reduced crossed products of simple $C^{*}$ algebras, Comm. Math. Phys. 81 (1981), 429-435.

[7] W. Krieger, On non-singular transformations of a measure space, I, II, Z. Wahrsch. Th. 11 (1969), 83-97; 98-119.

[8] W. Krieger, On ergodic flows and isomorphism of factors, Math. Ann. 223 (1976), $19-70$.

[9] G. Liu and F. Li, On Strongly Graded Rings and the Corresponding Clifford Theorem, Algebra Colloq. 13 (2006), 181-196.

[10] P. Lundström, Separable Groupoid Rings, Comm. Algebra 34 (2006), 3029-3041.

[11] J. R. Munkres, Topology, Second Edition, Prentice-Hall, 2000.

[12] F. J. Murray and J. von Neumann, On rings of operators, Ann. of Math. 37 (1936), $116-229$.

[13] F. J. Murray and J. von Neumann, On rings of operators IV, Ann. of Math. 44 (1943), 716-808.

[14] J. von Neumann, Collected works vol. III. Rings of operators, Pergamon Press, 1961.

[15] J. Öinert and P. Lundström, Commutativity and Ideals in Category Crossed Products, Proc. Est. Acad. Sci. 59(4) (2010), 338-346.

[16] J. Öinert and P. Lundström, The Ideal Intersection Property for Groupoid Graded Rings, accepted for publication in Comm. Algebra. Preprint available at arXiv:1001.0303v3 [math.RA]

[17] G. K. Pedersen, $C^{*}$-algebras and their Automorphism Groups, Academic Press, 1979.

[18] J. C. Quigg and J. S. Spielberg, Regularity and hyporegularity in $C^{*}$-dynamical system, Houston J. Math. 18 (1992), 139-152.

[19] J. S. Spielberg, Free-product groups, Cuntz-Krieger algebras, and covariant maps, Internat. J. Math. 2 (1991), 457-476.

[20] C. Svensson, S. Silvestrov and M. de Jeu, Dynamical Systems and Commutants in Crossed Products, Internat. J. Math. 18(4) (2007), 455-471. 


\section{References}

[21] C. Svensson, S. Silvestrov and M. de Jeu, Connections between dynamical systems and crossed products of Banach algebras by $\mathbb{Z}$, in Methods of Spectral Analysis in Mathematical Physics, Operator Theory: Advances and Applications, Vol. 186, Birkhauser, 2009, pp. 391-402.

[22] C. Svensson, S. Silvestrov and M. de Jeu, Dynamical Systems Associated with Crossed Products, Acta Appl. Math. 108 (2009), 547-559.

[23] J. Tomiyama, The interplay between topological dynamics and theory of $C^{*}$ algebras. Lecture Notes Series, 2. Global Anal. Research Center, Seoul, 1992.

[24] G. Zeller-Meier, Produits croisés d'une $C^{*}$-algèbre par un groupe d'automorphismes, J. Math. pures et appl. 47 (1968), 101-239. 